\title{
Model-Based Quality Estimation of Fingerprint Images
}

\author{
Sanghoon Lee, Chulhan Lee, and Jaihie Kim \\ Biometrics Engineering Research Center(BERC), \\ Department of Electrical and Electronic Engineering, Yonsei University, Seoul, Korea
}

\{hoony, devices, jhkim\}@yonsei.ac.kr

\begin{abstract}
Most automatic fingerprint identification systems identify a person using minutiae. However, minutiae depend almost entirely on the quality of the fingerprint images that are captured. Therefore, it is important that the matching step uses only reliable minutiae. The quality estimation algorithm deduces the availability of the extracted minutiae and allows for a matching step that will use only reliable minutiae. We propose a model-based quality estimation of fingerprint images. We assume that the ideal structure of a fingerprint image takes the shape of a sinusoidal wave consisting of ridges and valleys. To determine the quality of a fingerprint image, the similarity between the sinusoidal wave and the input fingerprint image is measured. The proposed method uses the 1-dimensional (1D) probability density function (PDF) obtained by projecting the 2-dimensional (2D) gradient vectors of the ridges and valleys in the orthogonal direction to the local ridge orientation. Quality measurement is then caculated as the similarity between the 1D probability density functions of the sinusoidal wave and the input fingerprint image. In our experiments, we compared the proposed method and other conventional methods using FVC-2002 DB I, III procedures. The performance of verification and the separability between good and bad regions were tested.
\end{abstract}

\section{Introduction}

The performance of any fingerprint recognition system is very sensitive to the quality of the acquired fingerprint images. There are three factors that lead to poor quality fingerprint images: 1) Physical skin injuries: scratches, broken ridges, and abrasions, 2) Circumstantial influences: wet or dry levels of humidity and dirty fingers, 3) Inconsistent contact: excessive or weak pressure. There are many previous works that deal with estimating the quality of fingerprint images. Hong et al. [1 modeled the ridge and valley pattern as a sinusoidal wave, and calculated amplitude, frequency and variance to determine the quality of fingerprint images. Michael [2] computed the mean and the variance of a sub-block of fingerprint images to measure the quality. Neither method was able to distinctly classify good regions and bad regions within the images. Bolle et al. 3 proposed a method that used the ratio of the directional region to the non-directional region. However, a limitation of this method is that the gray-level ridge and valley 
structure of fingerprint images contains much more information. Shen, et al. 4] used the variance of the 8-directional Gabor filter response. The performance of this method depends on the number of Gabor filters, and the computational complexity is high. Ratha and Bolle [5] proposed a method for image quality estimation in the wavelet domain, which is suitable for WSQ-compressed fingerprint images. But it is unsuitable when dealing with uncompressed fingerprint images. Lim [6] observed both global uniformity and local texture patterns in fingerprint images. However, it is necessary to determine the weights for global and local quality measurements when using this method. In this paper, we propose model-based quality estimation of fingerprint images. The structure of an ideal fingerprint image takes the shape of a sinusoidal wave. To determine the quality of each sub-block image, we measure the similarity between the ideal fingerprint structure (sinusoidal wave) and the input fingerprint structure. In the following sections, we will explain model-based quality estimation of fingerprint images. Section 2 addresses the main steps of our algorithm and the method used to measure the similarity between the ideal fingerprint structure and the input fingerprint image. In section 3, the proposed method is compared to previous methods using the separability between good and bad regions and the performance of fingerprint verification. Section 4 shows the conclusions we arrived at in the course of our experiments.

\section{Model-Based Quality Estimation}

Fingerprint quality estimation divides a pixel (or a block) in an input fingerprint image into good regions and bad regions. Good regions are the regions where minutiae can be detected. Bad regions are the regions where minutiae cannot be detected or false minutiae are more prominent. The ideal fingerprint region can be shown by a mono-dimensional sinusoidal wave and the obscure region is represented by an arbitrary wave. The main idea of our proposed method is to measure the similarity of the structures between the sinusoidal wave and the input fingerprint image. This method is inspired by independent component analysis (ICA) that extracts a 1-dimensional independent signal from n-dimensional mixture signals [7]. Fig. 1] shows the overall procedure of our proposed method schematically.

\subsection{Preprocessing}

The preprocessing stage that is composed of normalization and Gaussian masking. We used normalization and Gaussian smoothing to remove the effects of sensor noise and finger pressure difference.

\subsection{D-Gradient Vectors}

2D-gradient vectors of fingerprint images are obtained by gradient operators. Depending on computational requirements, either the Prewitt operator, the Sobel 


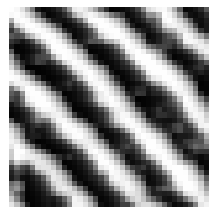

(a)

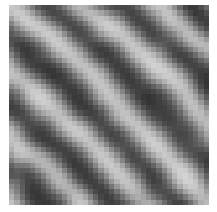

(b)

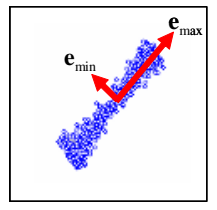

(c)

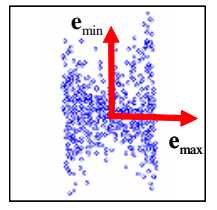

(d)

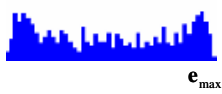

(e)

Fig. 1. Quality measurement block diagram: (a) Sub-block fingerprint image; (b) Preprocessing; (c) 2D-Gradient vectors; (d) Whitening; (e) 1D-Gradient PDF

operator, or the Marr-Hildreth operator [8] is chosen. In this paper, we used the Sobel operator. Fig. 1(c) shows the 2-channel gradient of a sub-block fingerprint image.

\subsection{Whitening}

Fig. 1(c) shows the 2D-gradient vectors of a sub-block fingerprint image. The 2D-gradient vector mixes up the orthogonal and parallel differential information to the ridge orientation. Because only the orthogonal differential information to the ridge is required to acquire the $1 \mathrm{D}$-gradient $\mathrm{PDF}$ in order to estimate the quality of a sub-block of the fingerprint image, the mixed 2D-gradient vector must be separated. Fig. 1(d) indicates the whitened gradient vector that is rotated to align the horizontal axis $\left(\mathbf{e}_{\max }\right)$ in the orthogonal direction of the ridge orientation. The whitening process separates the mixed $2 \mathrm{D}$-gradient vector into two $1 \mathrm{D}$-gradient vectors: the gradient vector $G_{v}$ with only orthogonal differential information to the ridge orientation, and the gradient vector $G_{h}$ with only parallel differential information to the ridge orientation. Since we have separated the mixed 2D-gradient vector, we can obtain the 1D-gradient PDF(Fig. 1(d)) by projecting the whitened gradient vector $G_{v}$ to the $\mathbf{e}_{\max }$ axis.

\subsection{Quality Measurement}

In order to estimate the quality of the fingerprint image, we assume that the ideal structure of ridges and valleys shows a sinusoidal wave. At each sub-block of images, the 1D probability density function (PDF) is obtained by projecting the whitened 2D-gradient vectors in the orthogonal direction to the local ridge orientation. With finite samples, polynomial density expansion like Taylor expansion is used to estimate a PDF. However, two other expansions are usually used for PDF estimation: the Gram-Charlier expansion and the Edgeworth expansion. In this paper, we use the Gram-Charlier expansion with ChebyshevHermit polynomials to estimate the $1 \mathrm{D}$-gradient $\mathrm{PDF} p_{v}$ as follows:

$$
p_{v}(\xi) \approx \hat{p}_{v}(\xi)=\varphi(\xi)\left\{1+\kappa_{3}(v) \frac{\mathrm{H}_{3}(\xi)}{3 !}+\kappa_{4}(v) \frac{\mathrm{H}_{4}(\xi)}{4 !}\right\},
$$

where $\kappa_{3}$ and $\kappa_{4}$ are skewness and kurtosis, $H_{i}$ represents the Chebyshev-Hermit polynomials of order $\mathrm{i}$, and $\varphi(\xi)$ is the standardized Gaussian density. $\kappa_{3}$ is zero 
in the case of the variable $v$ with symmetric distributions. The entropy of the approximated density function is estimated as follows:

$$
H(v) \approx-\int \hat{p}_{v}(\xi) \log \hat{p}_{v}(\xi) d \xi=H\left(v_{\text {gauss }}\right)-\frac{\kappa_{4}^{2}(v)}{48},
$$

where $v_{\text {gauss }}$ is the Gaussian variable of the zero mean and unit variance. The following equation is explicitly derived:

$$
J(v)=H\left(v_{\text {gauss }}\right)-H(v) \propto \kappa_{4}^{2}(v),
$$

where $J(v)$ is negentropy [7. The 1D-gradient PDF of the ideal fingerprint region is sub-Gaussian and negentropy has a large value when the distribution of $v$ is sub-Gaussian. Therefore we may define the quality measurement as follows:

$$
\text { Quality }=\kappa_{4}^{2}(v) \approx J(v)
$$

However, $J(v)$ also has a large value when the distribution of $v$ is super-Gaussian. Because the 1D-gradient PDF of a dry or wet fingerprint region is superGaussian, the quality measurement must discriminate between images that are sub-Gaussian and super-Gaussian. Therefore, the quality measurement defined in equation (6) must be adjusted as follows:

$$
\text { Quality }=\operatorname{sign}\left(\kappa_{4}(v)\right) \kappa_{4}^{2}(v)
$$

Because expectations of polynomials like the fourth power $\left(\kappa_{4}(v)=E\left\{v^{4}\right\}-3\right)$ are much more strongly affected by data far from zero than by data close to zero, approximation kurtosis by a non-polynomial function $\mathrm{G}$ is used [7]:

$$
\begin{aligned}
& \kappa_{4}(v)=\mathrm{E}\{\mathrm{G}(v)\}-\mathrm{E}\left\{\mathrm{G}\left(v_{\text {gauss }}\right)\right\} \\
& \mathrm{G}(v)=\frac{1}{\mathrm{a}} \log (\cosh (\mathrm{a} v)), 1 \leq \mathrm{a} \leq 2
\end{aligned}
$$

\section{Experimental Results}

The quality value procedure assigned validity to each $8 \times 8$ block and quantized 256 levels (with 255 the highest quality and 0 the lowest). Fig. 2(a) is a sample fingerprint image that includes a region of interest (ridges and valleys) and a background region. The block-wise quality value for the fingerprint image in Fig. 2(a) is shown in Fig. 2(b).

\subsection{Separability of Quality Measurement: Separability Between High and Poor Quality Regions}

We evaluated the proposed quality measurement using separability between values from good and bad regions. We first defined the quality of the sub-block by including minutiae as good and bad regions. The good regions are the sub-blocks around 


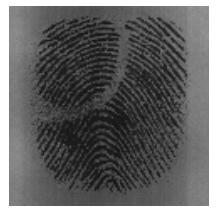

(a)

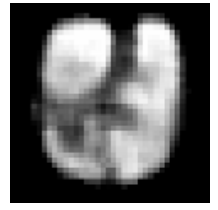

(b)

Fig. 2. Quantized quality value: (a) Original image; (b) Block-wise quality value

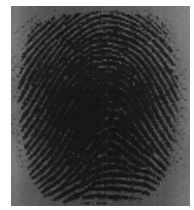

(a)

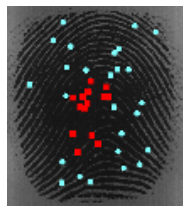

(b)

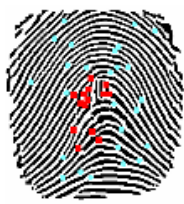

(c)

Fig. 3. Minutiae points of manually-defined quality (false minutiae: red rectangles, true minutiae: blue circles): (a) Original image; (b) Enhanced binary image; (c) Marked Region

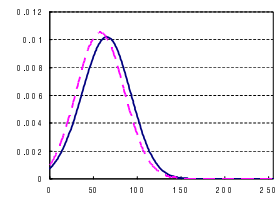

(a)

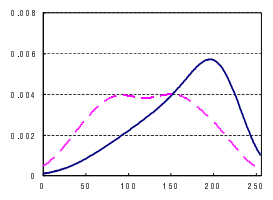

(b)

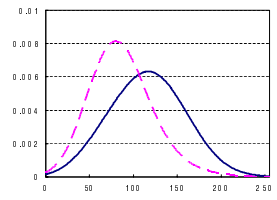

(c)

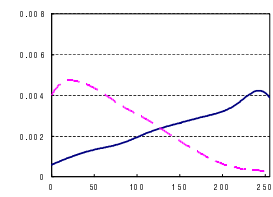

(d)

Fig. 4. Probability density function of each type of quality measurement (good region: solid line, bad region: dotted line): (a)Standard deviation; (b)Coherence; (c)Gabor; (d)The proposed method

the true minutiae and the bad regions are the sub-regions around the false minutiae. True minutiae are determined if the minutiae extracted by the feature extraction algorithm are equal to the manually extracted minutiae, and if the minutiae are not equal, we determined the minutiae as false minutiae. The proposed quality definition method is more objective than the visual (subjective) assessments method. Fig. 3 shows the true and false minutiae. With 100 randomly selected fingerprint images that were separated into good and bad regions, we calculated the probability distribution of each corresponding quality measurement. Fig. 4] shows the distribution of four quality measurements and Table 1 shows the separability of each distribution using FVC2002 DB I, III. These clearly show that the distribution when using the proposed method is more separable than when using existing methods. The separability is calculated as follows:

$$
\text { Separability }=\left|\mu_{\text {Good }}-\mu_{\text {Bad }}\right| / \sqrt{\left(\sigma_{\text {Good }}^{2}+\sigma_{\text {Bad }}^{2}\right)}
$$


Table 1. The separability of each type of quality measurement

\begin{tabular}{|c|c|c|}
\hline \multirow{2}{*}{ Quality Measurement } & \multicolumn{2}{|c|}{ Separability } \\
\cline { 2 - 3 } & DB I & DB III \\
\hline \hline Standard deviation & 0.19 & 0.05 \\
\hline Coherence & 0.64 & 0.88 \\
\hline Gabor filter & 0.61 & 0.44 \\
\hline Proposed method & 1.48 & 1.55 \\
\hline
\end{tabular}

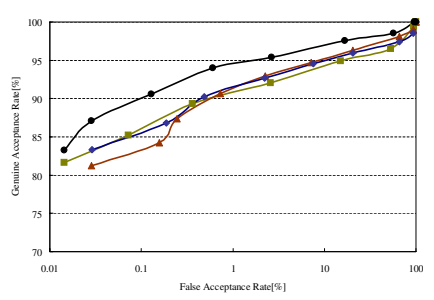

(a)

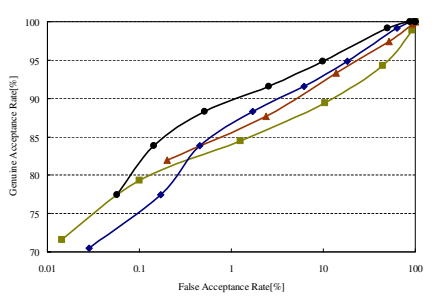

(b)

Fig. 5. Receiver Operating Curves (s.d. : rectangle, coherence : diamond, gabor : triangle, proposed method : circle) : (a) FVC 2002 DB I; (b) FVC 2002 DB III

\subsection{Verification Performance}

We examined verification performance according to the quality methods. The verification system used the same algorithms (preprocessing, frequency estimation [10, enhancement [1] and matching [11]) with the exception of the quality estimation algorithm. The thresholds for each quality estimation algorithm were chosen at the point of minimum quality decision error using a Bayesian decision. In the experiment, we compared the proposed method and other conventional methods using FVC-2002 DB I, III. Fig. 5 shows the matching results with the ROC in order to compare the proposed algorithm with existing algorithms. From this experiment, we can observe that performance of the fingerprint verification system was significantly improved when our quality estimation algorithm was applied to the input fingerprint images.

\section{Conclusions}

In this paper, we proposed a method to determine the quality of a fingerprint image with similarity between the ideal fingerprint model and an estimated 1DPDF. The ideal fingerprint image model has a monodimensional sinusoidal wave and uses a sub-Gaussian PDF when the project whitened 2D-gradient moves in the orthogonal direction of orientation of the sub-block. Quality estimation uses separability between high and poor quality regions and takes into account the performance of fingerprint verification. We compared the separability of each 
quality estimation method and the proposed method observed the highest separability using FVC-2002 DB I, III procedures. We also observed the lowest equal error rate (EER). The $1 \mathrm{D}-\mathrm{PDF}$ is influenced not only by the quality of the fingerprint image but also by the projection axis. The projection axis corresponds to the orientation of the sub-block in the fingerprint image. In further research, we will continue to examine the robust orientation estimation method.

\section{Acknowledgments}

This work was supported by the Korea Science and Engineering Foundation (KOSEF) through the Biometrics Engineering Research Center at Yonsei University.

\section{References}

1. L. Hong, Y. Wan and A. K. Jain, "Fingerprint Image Enhancement: Algorithm and Performance Evaluation", IEEE Transactions on Pattern Analysis and Machine Intelligence, vol.20, pp. 777 -789, Aug.1998

2. Michael Yi-Sheng Yao, Sharath Pankanti, Norman Haas, Nalini Ratha, Ruud M. Bolle, "Quantifying Quality: A Case Study in Fingerprints", AutoID'02 Proceedings Workshop on Automatic Identification Advanced Technologies, pp.126-131, March 2002.

3. Bolle et al, "System and method for determining the quality of fingerprint images", United State Patent number, US596956, 1999.

4. L. L. Shen, A. Kot and W.M. Koo, "Quality Measures of Fingerprint Images", Third International Conference on AVBPA 2001, pp. 266-271, Jun. 2001.

5. N. K. Ratha, and M. Bolle, "Fingerprint Image Quality Estimation", IBM Computer Science Research Report RC 21622, 1999.

6. Lim. E.,Jiang XD, Yau WY, "Fingerprint Quality and Validity Analysis", IEEE 2002 International Conference on Image Processing.

7. Aapo Hyvärinen, Juha Karhunen, Erkki Oja, "Independent Component Analysis", John Wiley Sons. Inc, 2001.

8. D.Marr, Vision. San Francisco, Calif.:W.H. Freeman, 1982.

9. Richard O.Dula, Peter E.Hart, David G.Stork, "Pattern Classification", John Wiley Sons. Inc, 2001.

10. Maio D., Maltoni D.," Ridge-line Density Estimation in Digital Images", International Conference on Pattern Recognition, Australia, August 1998.

11. D. Lee, K. Choi and Jaihie Kim, "A Robust Fingerprint Matching Algorithm Using Local Alignment", International Conference on Pattern Recognition, Quebec, Canada, August 2002. 